\title{
Transfusão de hemocomponentes em recém-nascidos de termo e prematuros
}

\author{
A.L. Albiero, E.M.A. Diniz, M.C.Z. Novaretti, F.A.C. Vaz, D.A.F. Chamone \\ Disciplinas de Hematologia-Hemoterapia e Pediatria Neonatal da Faculdade de Medicina da Universidade de São Paulo, São Paulo, SP.
}

RESUMO - Oвjetivo. Comparar o consumo de hemocomponentes entre recém-nascidos ( $R N)$ de termo (RNT) e pré-termo (RNPT) e correlacionar esse consumo ao ti po de tratamento dispensado à sua patologia: clínico ou cirúrgico; acidentes hemorrágicos e sobrevida.

Casuística e Metodologia. 48 rns classificados em dois grupos: 26 RNT e 22 RNPT receberam 251 unidades de hemocomponentes: 177 unidades de concentrado de hemácias (CH), 36 de concentrado de plaquetas (CP), 30 de plasma fresco congelado (PFC) e oi to de sangue total (ST), no período de 186 dias. Foi analisado o consumo de hemocomponentes em cada grupo, e na razão do número de RNs vivos por dia, até o 120 dia.

Resultados. O consumo médio de hemocomponentes foi de 7,31 unidades para RNPT e 3,46 para RNT. A análise de consumo diário revelou que a maior parte ocorreu em R Ns sob tratamento

\section{NTRODUÇÃO}

A prática transfusional em neonatologia tem sido objeto de interesse de muitos pesquisadores desde a década de 20 para tratar casos de desnutrição grave e de sepse em recém-nascidos (RNS) ${ }^{1}$, sobretudo em prematuros².

A fisiopatol ogia da doença hemolítica do recémnascido, DHRN (causada por anticorpos maternos contra o antígeno eritrocitário $D$ fetal) e seu tratamento estabeleceram-se nas décadas de 40 e $50^{3}$.

O aparelho circulatório dos RNS é um sistema aberto de pequenas dimensões, com características hemodinâmicas próprias, no qual a adição de pequenos volumes de hemocomponentes deve alcançar o melhor rendimento possível, com o mínimo de efeitos adversos: bioquímicos, imunológicos e infecciosos ${ }^{4-8}$.

Os RNS internados constituem o grupo de pacientes que, proporcionalmente, mais consome hemocomponentes em hospital. Esse consumo é inversamente proporcional ao seu peso e/ou idade gestacional $^{9}$. clínico antes do $60^{\circ}$ dia de vida (d.v.) e que um aumento após o $86^{\circ} \mathrm{d}$ d.v. pode ser atribuído a um aumento de cirurgias nessa fase. Os acidentes hemorrágicos predominaram em RNPT com plaquetometria inferior a $60.000 / \mathrm{mm}^{3}$. Foi constatada uma tendência inversamente proporcional entre o número de transfusões e a sobrevida.

Conclusões. Os RNPT consumiram mais hemocomponentes que os RNT. Esse consumo estava ligado à patologia de base. Foi sugerido que a transfusão profilática de CP em RNPT poderia reduzir o número de hemorragias, além do consumo de $\mathrm{CH}$ nesse grupo. Mais de dez transfusões de hemocomponentes nos primeiros 120 d.v., em ambos os grupos, parece constituir marcador de mau prognóstico.

UNITERMOS: Transfusão. Recém-nascido. Termo. Prematuro.

As razões pelas quais isso acontece são atribuídas à col eta excessiva de amostras de sangue do RN internado, à anemia tardia do prematuro ${ }^{10}$; mais raramente, à síndrome de produção insuficiente de eritrócitos, aos acidentes obstétricos e às malformações de placenta e de cordão ${ }^{11}$. A DHRN é a patologia que recebe o maior número de indi cações para exsangüineotransfusão ${ }^{12}$.

A transfusão de glóbul os vermelhos em RN pode ser indicada pelo melhor desempenho da hemoglobina A na hematose, por insuficiência respiratória ou por imaturidade do centro respiratório do tronco cerebral ${ }^{13}$.

A transfusão de plaquetas, segundo hemocomponente mais freqüentemente utilizado em $\mathrm{RNS}^{4,14,15}$, é indicada no tratamento das trombocitopenias específicas do RN, que podem ocorrer na fototerapia prolongada, nas infecções, na hipertensão pulmonar, na síndrome de aspiração meconial, na alo-imunização $\mathrm{Rh}$, na policitemia e em alguns distúrbios metabólicos ${ }^{16}$. São, ainda, indicadas transfusões de plaquetas em casos de destruição excessiva [coagulação intravascular disseminada, 


\begin{tabular}{|c|c|c|c|c|c|}
\hline & \multirow[t]{2}{*}{ Produtos } & \multicolumn{2}{|c|}{ Características } & \multicolumn{2}{|c|}{ Volume ${ }^{\star}(\mathrm{mL})$} \\
\hline & & & & $\begin{array}{c}\text { G-I } \\
\text { (RNT) }\end{array}$ & $\begin{array}{c}\text { G-II } \\
\text { (RNPT) }\end{array}$ \\
\hline $\mathrm{CH}$ & concentrado de hemácias & $\mathrm{Hb}: 50$ a $70 \mathrm{~g} / \mathrm{unidade}$ & HT: 60 a $80 \%$ & 40,90 & 23,01 \\
\hline $\mathrm{CP}$ & concentrado de plaquetas & $>5,5 \times 10^{10}$ plaqs. $/ \cup$ & $<1,0 \times 10,{ }^{8}$ leucs. $/ \bigcup$ & 57,00 & 45,32 \\
\hline PFC & plasma fresco congelado & Fator VIII: 50 a $150 \%$ & & 36,00 & 14,65 \\
\hline ST & sangue total & $\mathrm{Hb}: 50$ a $70 \mathrm{~g} / \mathrm{unidade}$ & & 455,00 & 326,70 \\
\hline
\end{tabular}

hemangiomas, trombocitopenia alo-imune neonatal (plaquetas maternas ou HLA idêntico), uso de próteses e de cateteres, e fármacos como penicilina, heparina, digoxina e ácido valpróico] e, mais raramente, de produção medular insuficiente e deficiências qualitativas congênitas (síndrome TAR, Fanconi, von Willebrand, Bernard-Soulier, Glanzmann etc. $)^{16}$.

Há fatores que dificultam a plena hemostasia dos RNS: sistema de coagulação imaturo, deficiência fisiológica dos fatores dependentes de vitamina $K$, capacidade significativamente diminuída de produzir trombina, maior fragilidade vascular ${ }^{16}$. Nessas situações, preconiza-se a transfusão de plasma fresco congelado.

Este trabal ho tem por objetivo comparar o consumo de sangue e hemocomponentes entre recémnascidos de termo e prematuros, analisar suas causas, correlacionando-as à sobrevida dos RNS e propor medidas que possam diminuir esse consumo.

\section{CASUÍSTICA E MÉTOdOS}

Características dos recém-nascidos - Estudamos as transfusões de hemocomponentes realizadas em 48 recém-nascidos internados na UCINE (Unidade de Cuidados Intensivos Neonatais para RN Externo) do Instituto da Criança "Prof. Pedro de Alcantara" do Hospital das Clínicas da F acul dade de Medicina da Universidade de São Paulo. Esta Unidade constitui um centro de referência para internação de RNS graves encaminhados de outros locais da cidade de São Paulo.

Os recém-nascidos têm idade inferior a 30 dias de vida ao ingressarem na UCINE. Como eles não são transferidos da Unidade até a sua recuperação, houve caso em que a primeira transfusão ocorreu no $51^{\circ}$ dia de vida e a última, no $120^{\circ}$ dia.

Eles foram classificados em dois grupos: RNS de termo (RNT), com 37 semanas ou mais, chamado de G-I, composto de 26 RNT e RNS pré-termo (RNPT), com tempo de gestação inferior a 37 semanas, chamado de G-II, composto de 22 RNPT.
A idade gestacional (IG) média ( $\pm D P$ ) do G-I foi de $39,02( \pm 1,08)$ semanas, e a do G-II, de 31,95 $( \pm 3,05)$ semanas. $O$ peso ao nascimento (PN) médio ( $\pm D P$ ), para o G-I , foi de $3.040( \pm 726) \mathrm{g}$ e, para o G-II , 1.642 $( \pm 704) g$.

A grande maioria dos RNS estudados era portadora de patologias graves. Eles foram agrupados segundo diagnóstico e tratamento em dois subgrupos: clínicos, com 35 pacientes (72,7\%), e cirúrgicos, com 13 pacientes (27,3\%).

Nos RNS sob tratamento clínico as patologias mais freqüentes foram: infecciosas (impetigo, onfalite, sepse e HIV); filhos de mãe com gravidez de alto risco: lúpica, diabética, bolsa rota por tempo prolongado (até dez dias), tentativa de abortamento, hemorragia intracraniana, gemelaridade e prematuridade extrema, deficiência de G-6PD, incompatibilidade materno-fetal (ABO e Rh), leucinose, malformações congênitas com tratamento clínico (sindrômicos, portadores de malformações óculo-auriculares, cardiopatias congênitas com insuficiência cardíaca), intoxicação digitálica, síndrome de Down com leucemia mielóide aguda e tumor congênito de adrenal em tratamento quimioterápico.

Nos RNS sob tratamento cirúrgico, os diagnósticos mais freqüentes foram: patologias de tubo digestivo (atresia congênita de esôfago, jejuno e íleo, enterite necrosante e volvo) e do trato urinário (válvula de uretra posterior e extrofia vesical), além de hérnia inguinoescrotal e umbilical.

Características dos produtos para transfusão As características dos produtos utilizados estão descritas na tabela 1 .

Todos os produtos utilizam o mei o CPDA-1 como solução anticoagulante/conservante. Todas as unidades de produtos celulares distribuídos aos RNPT foram irradiadas. Trinta unidades de $\mathrm{CH}$ (37\%) e as 18 unidades de CP (100\%) para RNT também foram irradiadas. As irradiações gama $(2,5 \mathrm{G} y)$ foram realizadas no mesmo dia, poucas horas antes das transfusões. 


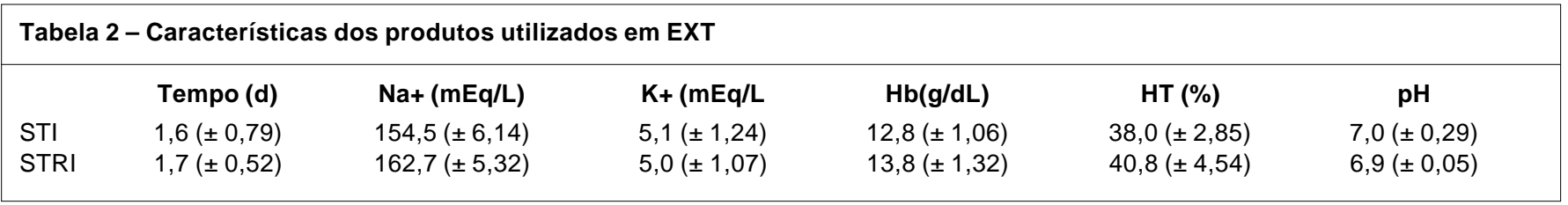

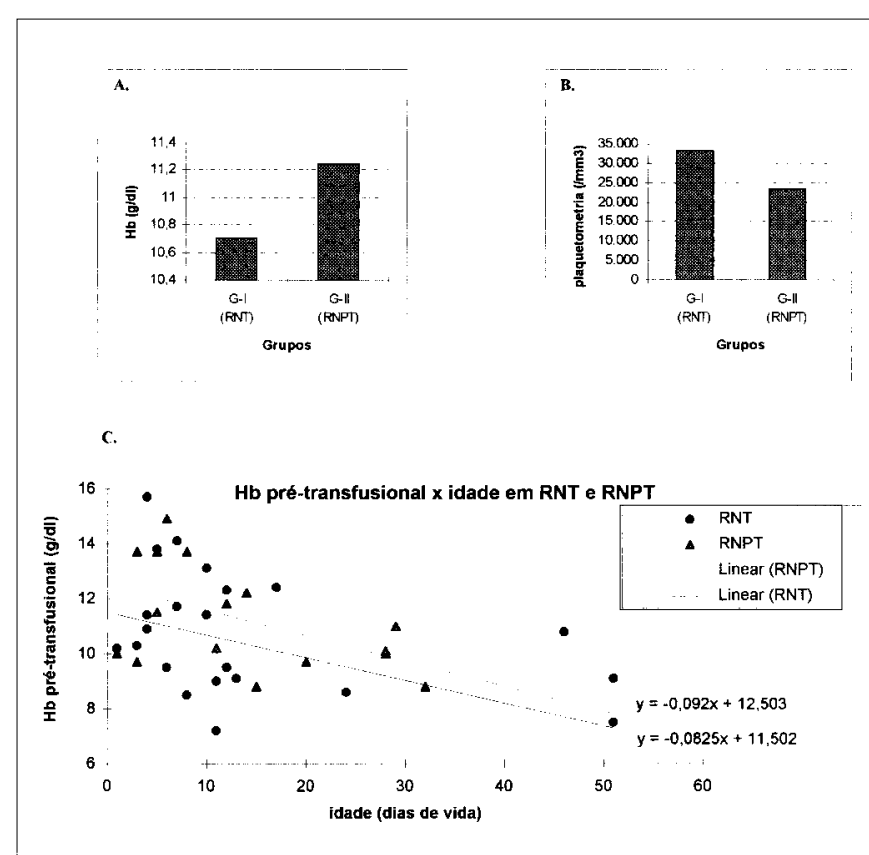

Gráfico 1 - Representa a média de dois parâmetros laboratoriais, A: nível sérico de hemoglobina ( $\mathrm{Hb})$; e $\mathrm{B}$ : plaquetometria, verificados imediatamente antes das transfusões de glóbulos e plaquetas, respectivamente para G-I (RNT) eG-II (RNPT). Em C, podemos observar a evol ução dos níveis de Hb prétransfusional de acordo com a idade (d.v.). A regressão linear, linha cheia, representa os RNT e a linha tracejada, os TNPT.

$\mathrm{Na}$ tabela 1, as duas últimas colunas referem-se a volumes que representam alíquotas de unidades maiores. E ssas alíquotas foram preparadas no banco de sangue, sem a abertura do sistema, utilizando-se o dispositivo de conexão estéril (SCD ${ }^{\mathrm{TM}} 312-$ HAEMONETICS) para a transferência dos produtos em bureta graduada com filtro de macroagregados interno. A reconstituição de sangue total a partir de $\mathrm{CH}$ e PFC também utilizou esse dispositivo.

Características dos produtos para exsangüineotransfusão (EXT) - A tabela 2 resume os resultados dos controles realizados nas unidades de sangue total irradiado (STI) e sangue total reconstituído ( $\mathrm{CH}+\mathrm{PFC}$ ) irradiado (STRI) com média e desviopadrão, entre parênteses, correspondentes.

A primeira col una da tabela 2 refere-se ao tempo de estocagem (em dias) da unidade utilizada para EXT desde a sua coleta. A irradiação foi sempre realizada poucas horas antes do procedimento.

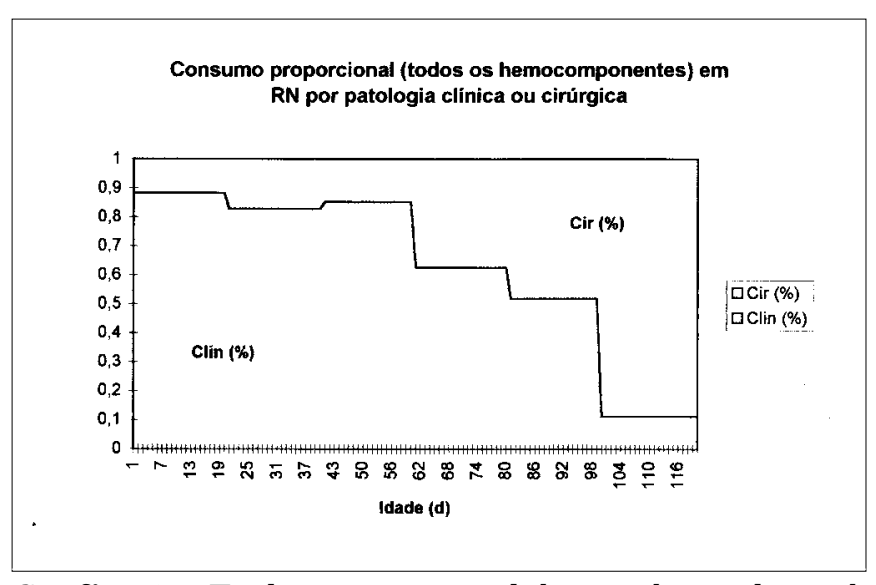

Gráfico 2 - Evolução percentual do tipo de patol ogia de base: clínica (Clin) ou cirúrgica (Cir), ao longo dos dias de vida dos RN que receberam transfusão.

Indicação das transfusões - As indicações de transfusão de hemocomponentes foram feitas de acordo com a rotina da UCINE baseadas em critérios clínicos e o banco de sangue atendeu a todas as solicitações. Apenas dois dos parâmetros laboratoriais utilizados para a indicação de transfusão de componentes globulares e plaquetários foram utilizados retrospectivamente: a taxa de hemoglobina sérica e a plaquetometria.

Por definição, transfusão profilática de plaquetas é aquela indicada baseada somente pela plaquetopenia e terapêutica, quando há sangramento em decorrência dessa plaquetopenia.

O nível médio de hemoglobina ( \pm DP) antes da transfusão de glóbulos nos recém-nascidos foi de $10,7( \pm 2,21) \mathrm{g} / \mathrm{dL}$ para RNT e $11,24( \pm 1,91) \mathrm{g} / \mathrm{dL}$ para RNPT $(p=0,374)$. A plaquetometria média ( $\pm D P)$ antes da transfusão de concentrado de plaquetas foi de $33.091( \pm 20.310) / \mathrm{mm}^{3}$ para RNT e 23.235 $( \pm 18.903) / \mathrm{mm}^{3}$ para RNPT $(p=0,091)$ [gráfico 1].

As indicações de transfusão de hemocomponentes, de acordo com o grupo de patol ogia de base ao qual o RN pertence, clínico ou cirúrgico, podem ser vistas no gráfico 2 .

O gráfico 2 mostra que cerca de $90 \%$ do consumo inicial de hemocomponentes ocorre em pacientes com patologias de tratamento clínico. Essa proporção mantém-se relativamente estável até por volta do 60 o dia de vida (d.v.), quando começa a ocorrer uma inversão nesse padrão. N o 86이. v. esse consu- 


\begin{tabular}{|cccccc|}
\hline \multicolumn{5}{|l|}{ Tabela 3 - Número de hemocomponentes utilizados por grupo de RN } \\
\hline \multicolumn{7}{|c}{ G-I (RNT) } & \multicolumn{2}{c|}{ G-II (RNPT) } \\
\cline { 5 - 6 } Componente & Unidades & U/RN & Unidades & U/RN & \\
CH & 62 & 2,38 & 115 & 5,22 & $\mathrm{p}=0,025$ \\
CP & 17 & 0,63 & 19 & 0,86 & $\mathrm{p}=0,682$ \\
PFC & 6 & 0,22 & 24 & 1,09 & $\mathrm{p}=0,006$ \\
ST & 5 & 0,19 & 3 & 0,13 & $\mathrm{p}=0,956$ \\
Total & 90 & 3,46 & 161 & 7,31 & $\mathrm{p}=0,026$ \\
\hline CH= concentrado de hemácias; CP= concentrado de plaquetas; PFC= plasma fresco congelado; ST= sangue total.
\end{tabular}

mo é cerca de $50 \%$ para RN com patologia clínica, e a outra metade, para paciente cirúrgico. No $120^{\circ}$ d.v., o consumo por paciente cirúrgico atinge o seu ápice (90\%) e o por paciente clínico, o seu mínimo (10\%).

\section{RESULTADOS}

Transfusões realizadas - No período de 186 dias, esses recém-nasci dos receberam 251 unidades de hemocomponentes, conforme ilustra a tabela 3.

A tabela 3 mostra o número de unidades transfundidas em cada grupo de RNS (unidades), por tipo de hemocomponente (concentrado de hemácias, concentrado de plaquetas, plasma fresco congelado e sangue total). Nas colunas "U/RN", vêemse as médias de unidades consumidas por indivíduo pertencente a cada grupo: RNT e RNPT, respectivamente.

I nício efreqüência das transfusões - A primeira transfusão (independentemente do componente) admi nistrada no grupo de 48 recém-nascidos ocorreu entre o 1o e o 51ㅇ dia de vida (média: 13 dias).

Apesar da média de idade no momento da primeira transfusão ter sido de 13 dias para todos os RNS e de não ter havido diferença entre G-I e G-II , $12 \%$ dos RNS receberam a primeira transfusão nas primeiras 24 horas de vida, 32\%, entre 2 e 7 dias, e $56 \%$, com mais de 7 dias.

A análise da freqüência das demais transfusões (após a primeira) até o $120^{\circ}$ dia de vida foi feita através de uma curva de regressão polinomial, onde se verifica a rel ação entre o número de produtos transfundidos e o de crianças vivas no mesmo dia. O gráfico 3 mostra essas curvas.

A análise dessas curvas mostra que de fato, de uma maneira geral, o consumo de hemocomponentes por RNPT é maior que o por RNT, exceto o consumo de plaquetas, que nos primeiros 12 dias de vida foi maior pelos RNT.

Esse consumo tende a declinar após o 30 d.v., atingindo o mínimo na faixa etária compreendida entre o 70 e o 100 d.v., quando o consumo de

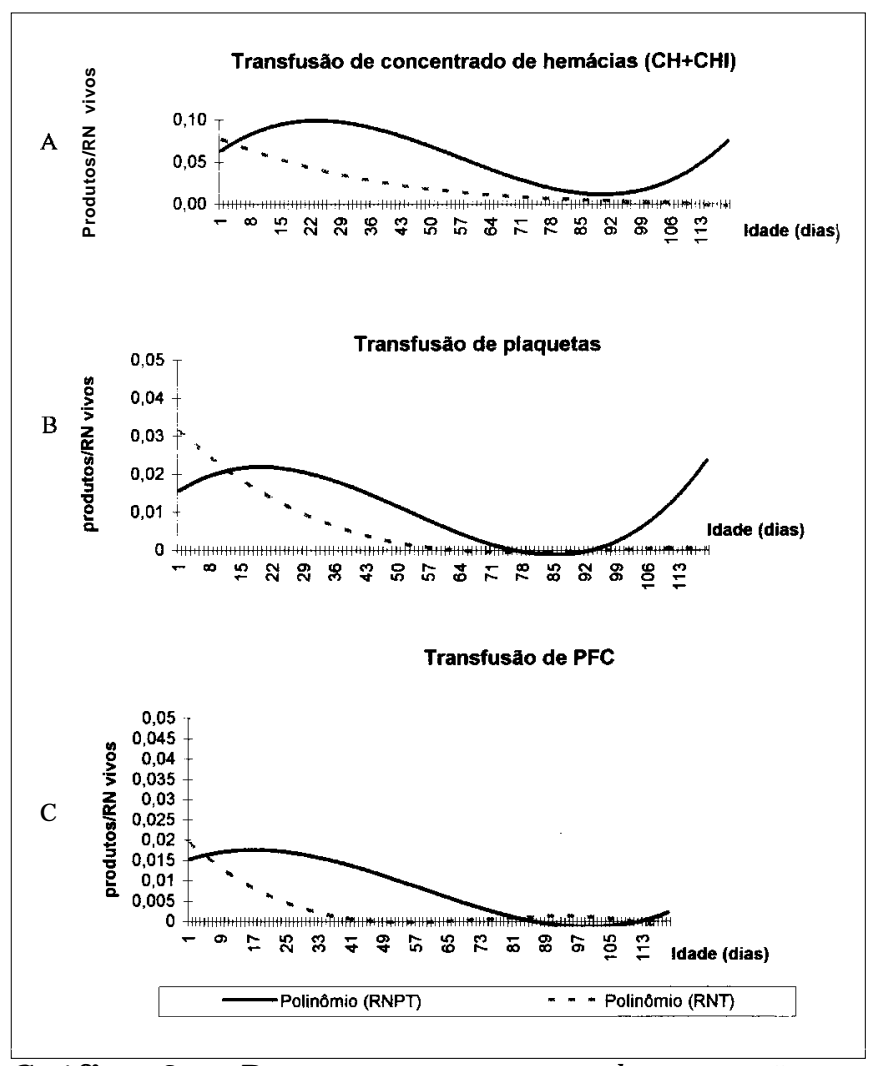

Gráfico 3 - Representa as curvas de regressão polinomial de grau 3 que relaciona o número de transfusões de unidades de hemácias (A), plaquetas (B) e plasma fresco congelado (C) ao número de recém-nascidos de termo (RNT) e prematuros (RNPT) vivos no mesmo dia.

hemácias e plaquetas para RNPT voltou a crescer. Esse aumento de consumo nesse período coincide com o de predomínio de patologias cirúrgicas (ver gráfico 2).

O intervalo médio entre duas transfusões de concentrado de hemácias para RNT foi de 8,38 dias, num período médio entre a primeira e a última transfusão de 23,10 dias. Para os RNPT, esse intervalo foi menor: 5,68 dias, e o período entre a primeira e a última transfusão é maior: 28,80 dias $(p=0,001)$. Para concentrado de plaquetas, o inter- 
valo médio entre duas transfusões para os RNT foi de 3,00 dias e para os RNPT, 3,67 dias $(p=0,304)$. Para plasma fresco congelado, 1,71 dia para RNT e 6,30 dias para RNPT $(p<0,001)$.

Exsangüineotransfusões (EXT) - Foram realizadas nove exsangüineotransfusões no período, em oito recém-nascidos: três RNPT e cinco RNT. Um deles foi submetido ao procedimento duas vezes $(p=0,623)$.

Três dos cinco RNT apresentavam incompatibilidade materno-fetal: um contra o antígeno A, um contra o antígeno $D$ e um contra os antígenos $C$ e $\mathrm{D}$ do sistema Rh. O quarto RNT tinha deficiência de G-6PD e evoluiu para óbito no 7o dia de vida. O quinto tinha um processo colestático, a bilirrubina conjugada era maior que a não-conjugada. Excluindo-se este último RN, a média de bilirrubina sérica não-conjugada pré-exsangüineotransfusão para os RNT foi de $30,1 \mathrm{mg} / \mathrm{dL}$.

Dentre oS RNPT que foram submetidos a EXT, um tinha atresia de esôfago, e dois, antecedente de rotura prematura de membranas (RPM). Um RN tinha atresia de jejuno, tendo falecido no $61^{\circ}$ d.v., e a outra criança fez exsangüineotransfusão no $21^{\circ}$ d.v. devido a sepse grave, tendo evoluído para óbito. A média de bilirrubina sérica não-conjugada pré-exsangüineotransfusão para OS RNPT foi de $21,7 \mathrm{mg} / \mathrm{dL}$.

Sobrevida - De todos os recém-nascidos estudados, constatamos que 17 evoluíram para óbito: 7 dos 26 RNT $(26,92 \%)$ e 10 dos 22 RNPT $(45,45 \%)$ [ $p=$ $0,523]$.

O gráfico 4 mostra duas curvas de sobrevida, num seguimento de 186 dias, a primeira, por grupo de RNS (RNT e RNPT) e, a segunda, por quantidade de transfusões recebi das. Verificamos que a sobrevida dos RNS estabilizou-se por vol ta do $100^{\circ}$ dia de vida: a dos RNT, em torno de $72 \%$ e a dos RNPT, por volta dos $57 \%$.

Com relação ao número de transfusões, a sobrevida de quem recebeu apenas uma unidade (14 indivíduos) foi de $93 \%$, de 2 a 9 transfusões (26 indivíduos), 73\% e acima de dez transfusões (10 indivíduos), apenas $10 \%$.

Dos 17 RNs que evoluíram para óbito, 85,71\% tinham patologia de base clínica e $14,28 \%$, de base cirúrgica. Dos 35 RNs do grupo clínico, 14 evoluíram para óbito: sobrevida de 60,0\%. Dos 13 RNs do grupo cirúrgico, três evoluíram para óbito: sobrevida de 76,92\% ( $p=0,654)$.

Houve 15 acidentes hemorrágicos diagnosticados em todos os RNS (31,25\%), 12 em RNPT (54,54\%). Destes, sete foram hemorragias intracranianas (HIC), três em trato urinário, um em trato gastrointestinal e um acidente de punção liquórica. Em
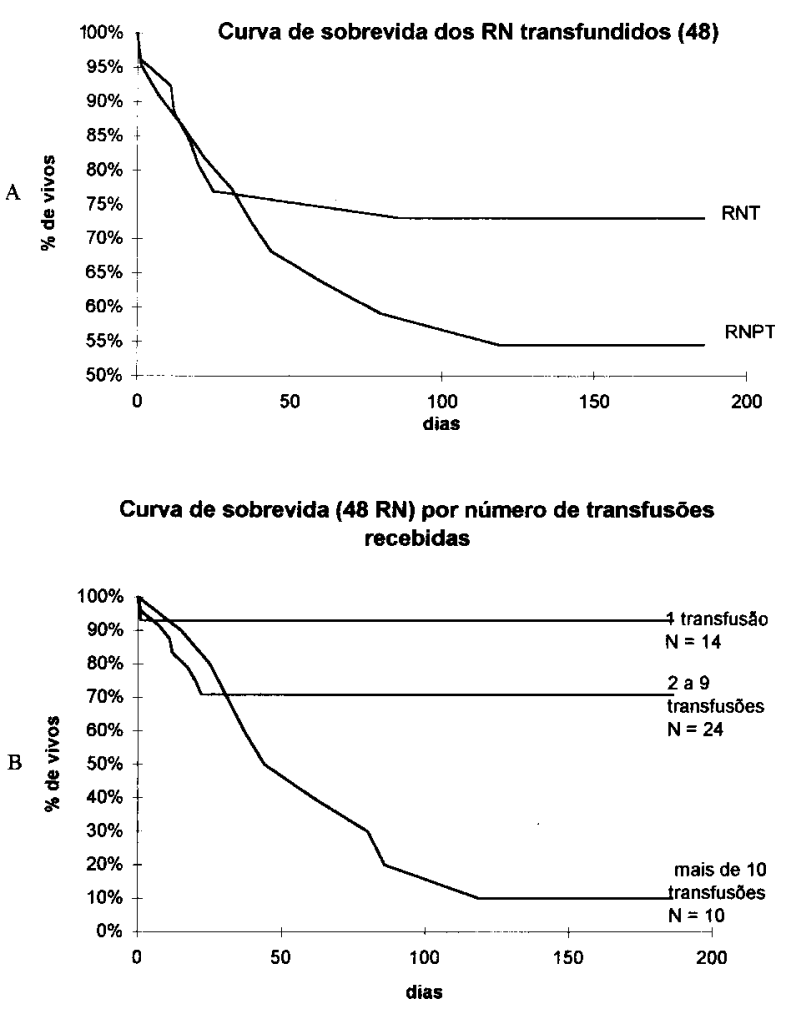

Gráfico 4 - Curvas de sobrevida dos recém-nascidos que receberam transfusão de hemocomponentes: (A), segundo a idade gestacional ao nascimento (RNT e RNPT) e (B), segundo o número de transfusões recebidas.

14/15 acidentes, foram observadas contagens plaquetárias inferiores a 60.000 plaqs. $/ \mathrm{mm}^{3}$, todas as HIC foram observadas em prematuros com menos de 34 semanas de IG ao nascimento, seis delas $(85,71 \%)$ associadas a plaquetometria inferior a 60.000 plaqs. $/ \mathrm{mm}^{3}$ (gráfico 5).

\section{DISCUSSÃO}

Além dos níveis de hemogl obina/hematócrito, os parâmetros mais constantemente relatados em literatura para indicar transfusão de hemácias e plaquetas em recém-nascidos são: risco associado (complicações respiratórias e/ou infecciosas), prematuridade extrema (idade gestacional inferior a 33 semanas) e idade (dias de vida) 4,14,17,18.

E m breve revisão da literatura, não constatamos relatos considerando esses três parâmetros simultaneamente. Ainda entre os Serviços que utilizam um ou dois deles, não há consenso entre os limites de hemoglobina/hematócrito que indicam a transfusão. As variações desses limites são grandes, mesmo de acordo com o tipo de institui- 
ção: hospital-escola, instituição pública, instituição privada?.

O quadro 1 procura sintetizar os três parâmetros mais freqüentemente mencionados e sugerir os limites de $\mathrm{Hb}$ e plaquetometria mais próximos do consenso para indi car transfusão de hemácias e plaquetas, respectivamente.

O gráfico 1-C mostra que o neonatologista preocupa-se em transfundir hemácias em RNs com níveis de hemoglobina tanto mais el evados quanto menor a sua idade (pós-natal) e, também, quanto menor sua idade gestacional. Esse cuidado adicional com os RNPT persiste ao longo dos dias de sobrevida (representada pelo segmento tracejado do gráfico quase paralelo ao segmento cheio). Esses segmentos representam as curvas de regressão linear dos pontos do gráfico que marcam os níveis de hemoglobina pré-transfusional para RNT e RNPT.

Os níveis de hemoglobina $(\mathrm{H} b)$ sérica pré-transfusão de hemácias são mais aproximados à recomendação da literatura que a plaquetometria observada antes da transfusão de plaquetas ${ }^{15,16,19}$. Os níveis de $\mathrm{Hb}$ pré-transfusão de hemácias para RNPT foram, em média, 1,0g/dL maior que para RNT. Ao contrário, a plaquetometria que indicou transfusão de concentrados de plaquetas (CP) para RNPT foi, na média, menor que para RNT (gráfico 1B). Essas diferenças não foram estatisticamente significativas.

I sso indica que os níveis séricos de $\mathrm{Hb}$ e plaquetometria não foram considerados parâmetros com a mesma importância para indicar a transfusão de hemácias e plaquetas, respectivamente.

$\mathrm{O}$ consumo de hemácias $(\mathrm{CH})$ e plasma $(\mathrm{PFC})$ foi estatisticamente diferente entre RNT e RNPT $(p=0,025$ e $p=0,006$, respectivamente), mas essa diferença não se observou no consumo de plaquetas $(p=0,682)$ [tabela 3].

Os acidentes hemorrágicos predominaram entre oS RNPT com plaquetometria inferior a 60.000/ $\mathrm{mm}^{3}$ (gráfico 5).

O coeficiente de variabilidade (cv) dos métodos para contagem de plaquetas é de $4 \%$ para os métodos de contagem el etrônica automatizada, $11 \%$ a 16\% para a contagem em câmara (hemocitômetro) $)^{20}$ e maior que isso para o método de contagem indireta (F ônio). O cV é sistemático e incide, igual mente, sobre ambos os grupos.

Durante o período consi der ado em nosso estudo, o método utilizado para contagem de plaquetas foi o automatizado: "Cell-Dyn 3.000" com CV de 4\%.

A relação entre os benefícios da transfusão profilática de plaquetas para a proteção contra sangramentos espontâneos em recém-nascidos e os riscos inerentes a essas transfusões (ex.: trans-

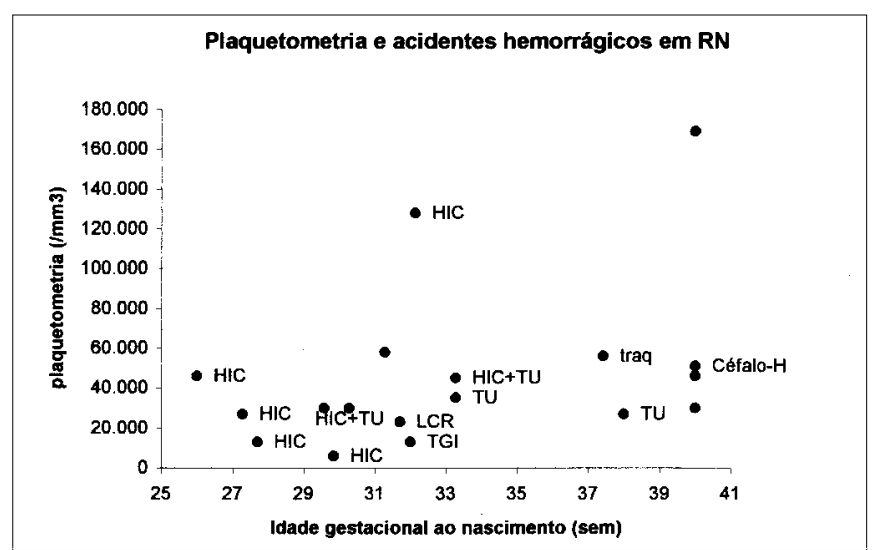

Gráfico 5 - Representa os acidentes hemorrágicos (HIC=hemorragia intracraniana, TU=trato urinário, TGI =trato gastrointestinal, LCR =líquor, traq=traqueal, Céfalo-H =céfalo-hematoma) verificados nos RNs segundo sua idade gestacional ao nascimento, em semanas, e respectivas plaquetometrias, por $\mathrm{mm}^{3}$.

missão de vírus) ainda não está bem estabel ecida.

Além da vantagem da proteção contra acidentes hemorrágicos espontâneos, a transfusão profilática de plaquetas poderia causar uma diminuição na necessidade transfusional de hemácias, o que implicaria em um número de doadores resultante não diferente daquele ao qual eles já estariam expostos.

Mesmo admitindo que o limiar de plaquetometria para indicação da transfusão profilática de plaquetas em recém-nascidos ainda não esteja bem definido na literatura, a associação americana dos bancos de sangue (AABB - American Association of Blood Banks) recomenda que esta deva ser feita aos $50.000 / \mathrm{mm}^{3}$ para prematuros sem riscos associados e $100.000 / \mathrm{mm}^{3}$ para os prematuros com riscos associados (ex. infecções).

A pesar da recomendação da AABB, alguns autores $^{19}$ propõem que a transfusão profilática de plaquetas seja feita com uma contagem em torno de $60.000 / \mathrm{mm}^{3}$ em prematuros com risco associado, e a grande maioria dos estabel ecimentos norte-americanos $^{7}$ estratifica empiricamente a indicação em função da prematuridade e dos riscos associados.

A indicação inequívoca de irradiação gama de componentes celulares em RN, para a profilaxia da reação enxerto-versus-hospedeiro pós-transfusional, restringe-se às transfusões específicas de doadores diretamente relacionados (pais e irmãos), à exsangüineotransfusão e à prematuridade. Esta última indicação, na literatura, alterna-se com a de peso inferior a $1.200 \mathrm{~g}$. Logo, mais uma vez, os autores devem estar referindo-se à prematuridade extrema. Outras indicações são controversas ${ }^{3,21}$. 
Quadro 1 - Indicação de transfusão de hemácias e profilática de plaquetas em recém-nascidos

\begin{tabular}{|c|c|c|c|c|}
\hline Quadro clínico & Maturidade do RN & Idade & Hb prévio (g/dL) & Plaquetometria $\left(/ \mathrm{mm}^{3}\right)$ \\
\hline Risco associado: & & $\leq 7$ dias & 14,0 & \\
\hline Assist. resp. & $\mathrm{RN} \leq 37$ sem & $>7$ dias & 13,0 & 60.000 \\
\hline Oxigenoterapia & & $\leq 7$ dias & 13,0 & \\
\hline Infecção & $\mathrm{RN}>37$ sem & $>7$ dias & 12,0 & entre 20.000 e 40.000 \\
\hline \multirow{4}{*}{$\begin{array}{l}\text { Ausência de } \\
\text { risco associado }\end{array}$} & & $\leq 7$ dias & 12,0 & \\
\hline & $\mathrm{RN} \leq 37$ sem & $>7$ dias & 11,0 & entre 40.000 e 60.000 \\
\hline & & $\leq 7$ dias & 11,0 & \\
\hline & $\mathrm{RN}>37$ sem. & $>7$ dias & 10,0 & entre 10.000 e 20.000 \\
\hline
\end{tabular}

Com relação a exsangüineotransfusão, o número reduzido de procedimentos reflete uma tendência mundial, fruto do acompanhamento pré-natal, da profilaxia da sensibilização Rh e do diagnóstico e tratamento antenatal da doença hemolítica do recém-nascido (DHRN). A DHRN causada pelo antígeno D é uma doença que caminha para extinção nos países desenvolvidos. O uso de gamagl obulina endovenosa e antibioticoterapia apropriada também vem reduzindo bastante o número de indicações de EXT por sepse 22,23 .

As atresias congênitas, e outras formas de obstrução, com prejuízo do trânsito intestinal, aumentam a circulação êntero-hepática com reabsorção de bilirrubina não-conjugada ${ }^{12,23}$. Tivemos dois casos de hiperbilirrubinemia relacionada a descontinuidade do trato gastrointestinal que necessitaram de EXT.

Com relação às especificações requeridas aos produtos para $\mathrm{EXT}^{9}$, a tabela 3 mostra que tanto as unidades de STI quanto as de STRI estão apropriadas. Os níveis de $\mathrm{Na}^{+}$e $\mathrm{Hb}$ dos STRI são discretamente mais elevados que os dos STI.

A diferença encontrada entre os volumes de produtos distribuídos para os dois grupos (RNT e RNPT) [tabela 1] é proporcional à diferença do peso médio dos RNS de cada grupo, logo, pela relação volume transfundido/peso, não houve diferença estatística entre os grupos.

De uma maneira geral, o consumo de hemocomponentes pelos RNPT foi cerca de $60 \%$ maior que o pelos RNT. A maior necessidade de transfusão em RNPT ocorre devido à exacerbação das complicações presentes nesse grupo de RNs. Eles estão sujeitos a mais complicações infecciosas e respiratórias que os RNT. Sua hemostasia é mais frágil e a porcentagem de $\mathrm{Hb}$ fetal é maior que nos RNT, piorando a hematose.

O padrão de produção de eritropoi etina no RNPT ainda é hepático, como na vida fetal, e, portanto, de um feedback menos sensível à hipoxemia que a eritropoietina produzida pelos rins (RNT e adul-

\begin{tabular}{|c|c|c|}
\hline \multicolumn{3}{|c|}{ Quadro 2 - Exposição a doadores diferentes } \\
\hline $\begin{array}{r}\text { Núm } \\
\text { ex }\end{array}$ & $\begin{array}{l}\text { nero de RNT } \\
\text { xpostos a }\end{array}$ & $\begin{array}{c}\text { Número de RNPT } \\
\text { expostos a }\end{array}$ \\
\hline 1 doador & 9 & 6 \\
\hline 2 doadores diferentes & 5 & 2 \\
\hline 3 doadores diferentes & 4 & 2 \\
\hline 4 ou 5 doadores diferentes & 3 & 2 \\
\hline 6 a 9 doadores diferentes & 4 & 3 \\
\hline 10 a 14 doadores diferentes & 1 & 3 \\
\hline 15 ou mais doadores diferentes & 0 & 4 \\
\hline Total & 26 & 22 \\
\hline
\end{tabular}

tos). A eritropoietina hepática protege o feto, que vive em hipoxemia, de policitemia e hiperviscosidade. Esse fenômeno explica satisfatoriamente a anemia tardia do prematuro ${ }^{24-27}$.

A anemia tardia do prematuro pode justificar em parte a diferença entre o consumo de hemácias por RNT e RNPT no período entre o $28^{\circ}$ e o $56^{\circ}$ dia de vida (gráfico 3-A). O uso da eritropoi etina humana recombinante ( $\mathrm{rH}$ u-EPO) em RNPT ${ }^{16,25,28}$ poderia reduzir a necessidade dos RNPT por transfusão de hemácias nesse período.

Cerca de $28 \%$ de todos os RNS receberam transfusão de apenas um hemocomponente. Entretanto, $62 \%$ receberam transfusão de mais de uma unidade de $\mathrm{CH}, 18 \%$, de mais de uma unidade de CP e $18 \%$, de mais de uma unidade de PFC.

Observando-se todos os produtos transfundidos em cada criança, podemos comparar o número de doadores diferentes a que se expõem os RNS de cada grupo.

Enquanto (21/26) $80,76 \%$ dos RNT foram expostos a um número de doadores menor ou igual a cinco, (10/22) 45,45\% dos RNPT o foram a um número de doadores maior que cinco, e (7/22) 31,81\%, a mais de dez doadores diferentes $(p>0,5)$.

A diferença entre a sobrevida dos RNT e RNPT pode ser observada no gráfico 3-A. U ma grande diferença na sobrevida também pode ser observa- 
da conforme o número de transfusões recebidas (gráfico 3-B): quanto maior o número de transfusões, menor a sobrevida.

Um efeito que deve ser levado em conta na proposta de diminuir a exposi ção dos RNS a diferentes doadores é o imunomodulador das transfusões. $\mathrm{Em}$ adultos, esse efeito tem sido freqüentemente relatado desde os anos 7029,30. Embora os recémnascidos pareçam não possuir sistema imunológico suficientemente desenvolvido para sofrer esse efeito ${ }^{31}$, há estudos demonstrando o contrário. Prematuros podem reagir contra antígenos do sistema HLA ${ }^{32}$, sistema intimamente implicado na imunomodulação ${ }^{29}$ e mais imunogênico que os sistemas antigênicos eritrocitários.

Outro procedimento que já vem sendo realizado também com o intui to de diminuir a exposição dos RNS a diferentes doadores é a aliquotagem das unidades.

Visto que o volume médio de uma unidade destinada a RNPT é $23 \mathrm{~mL}$, e que o intervalo médio entre duas transfusões é de 5,68 dias, uma única unidade de concentrado de hemácias, contendo $300 \mathrm{~mL}$, poderia atender a dois RNS simultaneamente, podendo ser aliquotado até seis vezes para cada RN, no seu período de validade de 35 dias.

As vantagens da menor exposição a doadores homólogos, do ponto de vista do risco de transmissão viral ${ }^{18,33-35}$, e aspectos imunol ógi cos superam os riscos relacionados a "lesões de estocagem" das hemácias, que, eventualmente, poderiam causar excesso de $\mathrm{K}^{+}$, hemoglobina livre e $\mathrm{pH}$ baix $0^{5,6}$.

\section{CONCLUSÕES}

- Consi deramos que o consumo mais el evado de hemocomponentes no grupo de RNPT parece ser uma conseqüência do maior número de complicações clínicas inerentes a esse grupo de pacientes.

- O principal grupo de patologias que justificou a necessidade transfusional após o 80 d.v. foi o cirúrgico, e a sobrevida desses pacientes nos 106 dias subseqüentes (até o 186ㅇ.v.) foi satisfatória (curvas de sobrevida estáveis após o 80 d.v.).

- As patologias que necessitaram de tratamento clínico justificaram a mai or parte do consumo de hemocomponentes antes do 80 d.v. e conferiram uma expectativa de vida pior aos RNS que as patologias cirúrgicas.

- A transfusão profilática de plaquetas poderia reduzir o número de acidentes hemorrágicos em prematuros e, com isso, reduzir a necessidade de transfusão de hemácias. A diminuição da morbidade relacionada a esses acidentes poderia produzir uma melhora na sobrevida.
- A transfusão de alíquotas reservadas a partir de uma única unidade de hemocomponente poderia reduzir a exposição a múltiplos doadores diferentes e, com isso, reduzir os riscos de transmissão viral e de sensibilização.

\section{SUMMARY}

\section{Blood transfusion in term and preterm infants}

BACKGROUND. Blood transfusion requirements for preterm infants are greater than for newborn ones. We compare blood transfusion requirements for newborn and premature infants and their pathology: clinical or surgical; hemorragic accidents and survival.

Methods. 48 newborns classified in 2 groups: 26 newborn and 22 preterm infants received 251 units of blood components: 177 units of red cell concentrates, 36 of platelet concentrates, 30 of fresh frozen plasma and 8 of total blood in a 186 days period. We analyzed total requirements of components in each group and daily, under a liveinfant/ day rate until 120 days.

Results. The all-components median requirements were 7.31 units for premature and 3.46 for newborn infants. Daily requirements analyzes reveal that requirements were greater before 60th day of life (d.I.) on clinical patients and after 86th d.I. may be caused by surgical acts. Hemorragic accidents happen on premature with less than 60.000 platel ets $/ \mathrm{mm}^{3}$. The survival wave by number of transfusions, until $186 \mathrm{~d} . \mathrm{l}$., shows an inversely proportional trend between the number of transfusions done and the hope of life.

Conclusions. Blood requirements for preterm infants are greater than for term ones. Those requirements are related to their pathology. Prophylatic platel et transfusions may reduce hemorrhagic accidents then red blood cell transfusions in preterm infants group. The number of transfusions over 10 is a surrogate marker of bad prognosis for both groups up to $120 \mathrm{~d}$.l. [Rev Ass Med Brasil 1998; 44(3): 201-9.]

KEY WORDS: Transfusion. Newborn infants. Premature infants.

\section{REFERÊ NCIAS BIBLIOGRÁFICAS}

1. Bakwin H, AstrowePS, Rivkin H. Transfused blood in newborns with severe malnutrition. AmJ Dis Child 1927; 33: 442-50.

2. Guéniot $P$, Séguy. Blood transfusion in premature infants. Gynéc Obstét 1925; 11: 111-8.

3. Fanaroff AA, Martin RJ . Neonatal-perinatal medicine. Diseases of thefetus and infant. 5th ed. St Louis, Mosby Year Book, 1992; 989-1.017. 
4. Chambers LA, I ssit LA. Supporting the pediatric transfusion recipient. Bethesda, American Association of Blood Banks, 1994.

5. Eshleman J R, Akinbi $\mathrm{H}$, Pleasure J et al. Prospective double bind study of small volumeneonatal transfusion with RBCs up to 35 days old. Transfusion 1994; 34(10S): 32S , S126.

6. Rodrigues J W, Mannino F, Lane T. Limitation of donor exposure in premature neonates and elimination of blood wastageusing novel transfusion strategy. Transfusion 1994; 34(10S): 32S, S125.

7. Strauss RG, Levy GJ , Sotello-Avila C et al. National survey of neonatal transfusion practices: II. Blood component therapy. Pediatrics 1993; 91(3): 530-6.

8. Tan KL, Wong LY. Effect of blood transfusion on acid base, glucose and electrolytic status in very low birth weight infants. Biol Neonate 1991; 59: 373-80.

9. Vaz FAC. Exsanguineotransfusão. In Santoro M, Diniz EMA. Manual deN eonatologia. Sociedade de Pediatria de São Paulo. Comitê de Neonatologia. Rio de J aneiro, Revinter, 1994.

10. Relier J P. Transfusion du nouveau-né. Gaz Transfusion 1993; 88: 4-16.

11. Oski, F.A. The erythrocyte and its disorders. In Nathan, DG, Oski, FA. Hematology of infancy and childhood. 4th ed. W.B. Saunders, 1993.

12. Ahlfors CE. Critera for exchange transfusion in jaundiced newborns. Pediatrics 1994; 93(3): 488-94.

13. Meyer J, Sive A, J acobs P. Empiric red cell transfusion in assyntomatic preterm infants. Acta Paediatr 1993; 82: 30-4.

14. Klemperer M. Perinatal and neonatal transfusion. I n Petz LD, Swisher SN. Clinical practiceof transfusion medicine. 2nd ed. New York, Churchil Livingstone, 1989; 615-34.

15. Strauss RA. Perinatal platel et and granulocyte transfusions. In Kennedy MS, Wilson S, Kelton J G (eds). Perinatal transfusion medicine. Arlington, American Association of Blood Banks, 1990; 123-44.

16. Beardsley DS. Platelet abnormalities in infancy and childhood. In Nathan \& Oski. Hematology and childhood. 4th ed, W.B. Saunders, 1993; 1.561-603.

17. Stockman III J A. Transfusions in the neonate. In Kennedy MS, Wilson S, Kelton J G (eds). Perinatal transfusion medicine. Arlington, American Association of Blood Banks, 1990; 103-21.

18. Strauss RG. Transfusion therapy in neonates. AmJ Dis Child 1991; 145: 904-11.

19. Andrew $M$, Vegh $P, C a c o C$ et al . A randomized, controlled trial of platelet transfusions in thrombocytopenic premature infants. J Pediatr 1993; 123(2): 285-91.

20. Todd-Sanford-Davidsohn. Diagnósticos clínicos e conduta terapêutica por exames Iaboratoriais. 16a ed. Ed. Manole. 1982; 969.
21. Shivdasani RA, Anderson KC. Graft-versus-host disease. In Petz LD, Swisher SN, Kleinman S et al. Clinical practice of transfusion medicine. 3th ed. New York, Churchil Livingstone, 1996; 931-46.

22. Ceccon MEJ, Diniz EMA, Ramos J LA, Vaz FAC. Exchange transfusion in newborn infants with perinatal hemolytic disease. Efficacy of the procedure. Rev Paul Med 1993; 111(2): 348-53.

23. Sharon BI. Transfusion therapy in congenital hemolytic anemias. Hemat/ Oncol Clin North Am 1994; 8(6): 1.053-86.

24. Brown MS, Garcia J F, Phibbs RH, Dallman PR. Decreased response of plasma immunoreactiveerythropoi etin to "available oxygen" in anemia of prematurity. J Pediatr 1984; 105: 793-8.

25. Chia YC, LeoneA. Eritropoi etina recombinante na anemia da prematuridade. Pediatria (São Paulo) 1995; 17(4): 174-90.

26. Stockman III J A, Graeber J E, Clark DA et al. Anemia of prematurity: determinants of the erythropoietin response. J Pedriatr 1984; 105: 786- 92.

27. Vengelen-Tyler $V$. editor-in-chief. Technical manual - American association of blood banks. 12th ed, Bethesda, 1996; 486.

28. Halperin DS, Wacker P, Lacourt G et al. Effects of recombinant human erythropoietin in infants with the anemia of prematurty: a pil ot study. J Pediatr 1990; 116: 779-86.

29. Andreu G. Transfusion et infections post-opértoires: revue et synthèse des recherches et de léxpérience clinique. Transfus Cient Biol 1994; 231-6.

30. Mincheff SM, Meryman HT, Kapoor $V$ et al. Blood transfusion and immunomodulation: a possiblemecanism. Vox Sang 1993; 65: 18-24.

31. Scornik J C, Pfaff WW, Howard RJ et al. Increased antibody responsiveness to blood transfusions in pediatric patients. Transplantation 1994; 58(12): 1.361-5.

32. Russel ARB, Rivers RPA, Davey N. The devel opment of antiHLA antibodies in multiply transfused preterm infants. Arch Dis Child 1993; 68: 49-51.

33. Liu EA, Mannino FL, Lane TA. Prospective, randomized trial of the safety and efficacy of a limited donor exposure transfusion program for premature neonates. J Pediatr 1994; 125(1): 92-6.

34. Luban NL, Strauss RG, HumeHA. Commentary on the safety of red cells preserved in extended-storage media for neonatal transfusions. Transfusion 1991; 31(3): 229-35.

35. Pouliquen A, Heshmati $F$, Brossard $Y$. Transfusion de globules rouges chez le nouveau-né: vers la reduction du nombre de donneurs et la transfusion de plus de 7 jours. In Progrès en neonatologie 13 (XXIII èmes J ournées National es en Néonatologie, 1993). Paris, Karger. 1993; 1: 68-76. 